\title{
NLO calculations matched with the Nagy-Soper parton shower
}

\author{
Institute for Theoretical Particle Physics and Cosmology \\ RWTH Aachen University \\ D-52056 Aachen, Germany \\ E-mails: \\ mczakon@physik.rwth-aachen. de \\ hartantoaphysik.rwth-aachen.de \\ krausephysik.rwth-aachen.de \\ worekephysik.rwth-aachen. de
}

Michal Czakon, Heribertus B. Hartanto, Manfred Kraus, Malgorzata Worek*

An MC@NLO-like matching of NLO QCD calculations with the Nagy-Soper parton shower is briefly summarised. Uncertainties and ambiguities of the matching scheme are shortly discussed. A few results for the $p p \rightarrow t \bar{t} j+X$ production process at the LHC with $\sqrt{s}=8 \mathrm{TeV}$ are also shown. All results have been obtained using the Nagy-Soper parton shower implementation in the DEDUCTOR program together with the HELAC-NLO framework.

12th International Symposium on Radiative Corrections (Radcor 2015) and LoopFest XIV (Radiative Corrections for the LHC and Future Colliders),

15-19 June 2015,

UCLA Department of Physics \& Astronomy Los Angeles, CA, USA

\footnotetext{
*Speaker.

${ }^{\dagger}$ Preprint number: TTK-16-04
} 


\section{Introduction}

After an almost two year shutdown, the Large Hadron Collider (LHC) started delivering physics data in 2015. This marked the begin of Run 2 at the LHC and opened the path to an even deeper understanding of the Standard Model (SM) physics and hopefully to new discoveries. Protons are now collided at the LHC at the record breaking energy of $13 \mathrm{TeV}$, almost double the collision energy of Run $1(\sqrt{s}=7,8 \mathrm{TeV})$. On the $15^{\text {th }}$ of December 2015 , the ATLAS and CMS experiments both reported a number of results using $\sqrt{s}=13 \mathrm{TeV}$ proton collision data. Even though the amount of data on which analyses are based is still limited, experimentalists have succeeded in producing numerous results for the SM physics, including new results for the Higgs boson and the top quark. Additionally, many searches for physics beyond the SM, yielding many improved limits beyond Run 1, have been presented. In 2016 we shall wait for more (and updated) results. To describe experimental data various theoretical tools are needed, among others, Monte Carlo (MC) generators that are widely used in all experimental analyses. Such programs need to be improved as data become more precise. There are various ways to reach better accuracy of theoretical predictions. In particular, one such possibility is an increase of the order in the fixed order perturbation expansion. Next-to-leading order (NLO) calculations can now be performed in a fully automatic manner. We mention here only the HELAC-NLO framework [1], that will be used in our studies. The software has recently been used to calculate NLO QCD corrections to a process with five objects in the final state, i.e. $W^{+} W^{-} b \bar{b} j$ [2]. Adding one more order complicates the picture tremendously. For next-to-next-to-leading order (NNLO) calculations, such automatic frameworks are not yet publicly available. In addition, a list of processes that have been completed at that order comprises processes with two objects in the final state only. The most prominent example being the NNLO calculation for the $t \bar{t}$ production process at hadron colliders, where two coloured and massive final state fermions are present [3,4]. Finally, an important milestone has been recently reached when a first calculation at the next-to-next-to-next-to-leading order $\left(\mathrm{N}^{3} \mathrm{LO}\right)$ in perturbative QCD for the Higgs production in gluon fusion in the large top-mass limit has been completed [5]. Since for each order one needs to include more Feynman diagrams both with one particle more in the final state and with one loop more in the intermediate state, a formal order-by-order perturbative calculation is limited to a few orders only. Alternatively, to improve the theoretical accuracy one can use parton shower (PS) programs. Parton shower algorithms approximate higher-order corrections by including the leading soft and collinear contributions to all orders. These programs are not able to correctly estimate the radiation of hard jets. They can, however, generate arbitrarily many jets in the final states. In order to improve the simulation of hard jets production in the parton shower, approaches were developed to match parton showers with fixed order NLO calculations $[6,7]$ and to merge matched calculations for different jet multiplicities both at LO and NLO [8-12]. Finally, those methods have already started to be applied to the NNLO calculations. Formally, however, in parton showers that are currently being use by experimental collaborations the resummation of soft and collinear contributions is only certified to Leading Logarithmic (LL) accuracy. In practice, many of the NLL contributions are already included, either by the angular ordering of the parton shower or by an optimal scale choice for $\alpha_{s}$. Thus, yet another progress on the front of theoretical predictions for the LHC can be accomplished by improving the parton shower itself. This can be done through an inclusion of color and spin correlations in subsequent parton shower emissions. 
An impact of the colour suppressed terms in parton shower simulations, for the final state parton shower, have already been studied in [13]. First results for the $e^{+} e^{-} \rightarrow 2 j$ production at LEP energy have shown that standard LEP-observables, e.g. event shapes and jet rates, are only slightly affected. However, for tailored observables, i.e. observables that are sensitive to soft (wide angle) splitting, deviations as large as $20 \%$ have been observed. The first step in the direction of the complete algorithm, which consistently takes into account both spin and color correlations in the parton emissions for the collision of hadrons, has been done by Nagy and Soper [14, 15]. A large part of their idea has been implemented in the DEDUCTOR program [16]. Even though the exact colour and spin correlations are not yet included in the software, it can already provide results with an extension of the leading colour approximation, which has been implemented instead.

In this proceedings we shall briefly summarise the concept behind the Nagy-Soper parton shower. In the next step we shall outline a matching procedure between the Nagy-Soper parton shower and a fixed order calculation at NLO [17]. To this end, we use the MC @ NLO approach [6]. When matching NLO calculations with parton shower programs, one needs to avoid the double counting of emissions, which on the one hand can be generated with a jet from the matrix element and on the other hand can appear as a jet from the parton shower. The Mc@ NLO method removes double counting contributions by expanding the parton shower to first order in $\alpha_{s}$ and compensating for the terms, which are already present at fixed order. Finally, a few results for the $p p \rightarrow t \bar{t} j$ production process, which involves non-trivial colour exchange, massive partons and requires cuts already at the Born level, will be shown.

\section{Nagy-Soper parton shower}

In case of a generic process $a+b \rightarrow m$ the expectation value for a completely inclusive observable $F$ in the Nagy-Soper formalism can be written as follows

$$
\sigma[F]=\sum_{m} \frac{1}{m !} \int\left[d\{p, f\}_{m}\right]\left\langle\mathscr{M}\left(\{p, f\}_{m}\right)\left|F\left(\{p, f\}_{m}\right)\right| \mathscr{M}\left(\{p, f\}_{m}\right)\right\rangle \frac{f_{a}\left(\eta_{a}, \mu_{F}^{2}\right) f_{b}\left(\eta_{b}, \mu_{F}^{2}\right)}{4 n_{c}(a) n_{c}(b) \times \text { flux }}
$$

where the sum runs over all final state multiplicities. Here, $\left[d\{p, f\}_{m}\right]$ is the sum of all $m$-particle phase space measures for different flavour sequences $\{f\}_{m}$. The factor $1 / m$ ! is necessary to account for identical contributions. The parton density functions evaluated at the momentum fraction $\eta$ and factorization scale $\mu_{F}^{2}$ are denoted by $f_{a / b}\left(\eta, \mu_{F}^{2}\right)$. The factor 4 in the denominator comes from averaging over initial state spins and $n_{c}(i)$ is the colour factor. The quantity

$$
\rho\left(\{p, f\}_{m}\right) \sim\left|\mathscr{M}\left(\{p, f\}_{m}\right)\right\rangle\left\langle\mathscr{M}\left(\{p, f\}_{m}\right)\right|,
$$

is the quantum density $\rho$ and a matrix element $\mathscr{M}$ can be viewed as a vector $\left|\mathscr{M}\left(\{p, f\}_{m}\right)\right\rangle$ in colour $\otimes$ spin space and can be resolved into basis vectors $\left|\{s\}_{m}\right\rangle$ and $\left|\{c\}_{m}\right\rangle$ with complex expansion coefficients $\mathscr{M}\left(\{p, f, s, c\}_{m}\right)$

$$
\left|\mathscr{M}\left(\{p, f\}_{m}\right)\right\rangle=\sum_{\{s\}_{m}} \sum_{\{c\}_{m}} \mathscr{M}\left(\{p, f, s, c\}_{m}\right)\left|\{s\}_{m}\right\rangle \otimes\left|\{c\}_{m}\right\rangle=\sum_{\{s, c\}_{m}} \mathscr{M}\left(\{p, f, s, c\}_{m}\right)\left|\{s, c\}_{m}\right\rangle .
$$

The propagation of the quantum density matrix $\rho$ from some initial shower time, $t_{0}$, to some final shower time, $t_{F}$, is described by the evolution equation. The initial shower time corresponds to 
the hard interaction, whereas the final shower time characterises the physical scale at which parton emissions cannot be described perturbatively. The perturbative evolution is described by a unitary operator $U\left(t_{F}, t_{0}\right)$ that obeys

$$
\frac{d U\left(t, t_{0}\right)}{d t}=\left[\mathscr{H}_{I}(t)-\mathscr{V}(t)\right] U\left(t, t_{0}\right) .
$$

Here, $\mathscr{H}_{I}(t)$ describes resolved emission and $\mathscr{V}(t)$ the unresolved/virtual one. The latter can be further decomposed into a color diagonal, $\mathscr{V}_{E}(t)$, and a color off-diagonal part, $\mathscr{V}_{S}(t)$, as follows

$$
\mathscr{V}(t)=\mathscr{V}_{E}(t)+\mathscr{V}_{S}(t)
$$

Eq. (2.4) can be solved as

$$
U\left(t, t_{0}\right)=N\left(t, t_{0}\right)+\int_{t_{0}}^{t} d \tau U(t, \tau)\left[\mathscr{H}_{I}(\tau)-\mathscr{V}_{S}(\tau)\right] N\left(\tau, t_{0}\right),
$$

where $N\left(t, t_{0}\right)$ is the Sudakov form factor (a number) defined as

$$
N\left(t, t_{0}\right)=\exp \left(-\int_{t_{0}}^{t} d \tau \mathscr{V}_{E}(\tau)\right)
$$

In case of a non-trivial colour evolution, the exponentiation of a non-diagonal matrix is very difficult. Thus, only the colour diagonal part, $\mathscr{V}_{E}(t)$, is exponentiated, whereas the off-diagonal part, $\mathscr{V}_{S}(t)$, is treated perturbatively in the same way as $\mathscr{H}_{I}(t)$. The expectation value of the observable $F$, including shower effects, is provided via

$$
\sigma[F]=\left(F \mid \rho\left(t_{F}\right)\right)=\left(F\left|U\left(t_{F}, t_{0}\right)\right| \rho\left(t_{0}\right)\right) .
$$

The evolution in the shower time is always ordered in some chosen kinematic variable to correctly resum leading logarithms of infrared sensitive quantities. In the Nagy-Soper parton shower the following variable has been proposed

$$
\Lambda_{l}^{2}=\frac{\left|\left(\hat{p}_{l} \pm \hat{p}_{m+1}\right)^{2}-m_{l}^{2}\right|}{2 p_{l} \cdot Q_{0}} Q_{0}^{2}, \quad \quad e^{-t}=\frac{\Lambda_{l}^{2}}{Q_{0}^{2}},
$$

where $\hat{p}_{l}$ is the emitter momentum after emission, $\hat{p}_{m+1}$ the emitted parton momentum, $p_{l}$ the emitter momentum before emission $\left(p_{l}^{2}=m_{l}^{2}\right)$ and $Q_{0}$ is the total final state momentum. The minus/plus sign between $\hat{p}_{l}$ and $\hat{p}_{m+1}$ in Eq. (2.9) applies to an initial/final state splitting.

\section{Matching Inclusive Processes}

There exist several schemes for matching NLO calculations with parton showers, the most popular being POWHEG and Mc@ NLO. In order to benefit from the recently implemented subtraction scheme based on the Nagy-Soper parton shower splitting kernels [18], which is implemented in the HelaC-Dipoles framework [19], we chose the Mc@NLO formalism. For a generic $a+b \rightarrow m$ process at NLO, one can write the quantum density matrix in a perturbative expansion in $\alpha_{s}$, according to

$$
\mid \rho)=\underbrace{\left.\mid \rho_{m}^{(0)}\right)}_{\text {Born, } \mathscr{O}(1)}+\underbrace{\left.\mid \rho_{m}^{(1)}\right)}_{\text {Virtual, } \mathscr{O}\left(\alpha_{s}\right)}+\underbrace{\left.\mid \rho_{m+1}^{(0)}\right)}_{\text {Real, } \mathscr{O}\left(\alpha_{s}\right)}+\mathscr{O}\left(\alpha_{s}^{2}\right),
$$


where the leading order contribution is counted as order 1 in the strong coupling $\alpha_{s}$. In addition, $\left.\mid \rho_{m}^{(0)}\right)$ and $\left.\mid \rho_{m+1}^{(0)}\right)$ are tree level matrix elements, whereas $\left.\mid \rho_{m}^{(1)}\right)$ is the one-loop amplitude. Based on this quantum density matrix, the expectation value of the observable $F$ including shower effects $\sigma[F]^{P S}=\left(F\left|U\left(t_{F}, t_{0}\right)\right| \rho\right)$ suffers from double counting. This can be seen when the iterative solution to the evolution equation is expanded to $\mathscr{O}\left(\alpha_{s}\right)$. Indeed, we obtain

$$
\left.\left.\left.\left.\mid \rho\left(t_{F}\right)\right)=U\left(t_{F}, t_{0}\right) \mid \rho\right) \approx \mid \rho\right)+\int_{t_{0}}^{t_{F}} d \tau\left[\mathscr{H}_{I}(\tau)-\mathscr{V}(\tau)\right] \mid \rho_{m}^{(0)}\right)+\mathscr{O}\left(\alpha_{s}^{2}\right)
$$

and $\left.\mid \rho\left(t_{F}\right)\right)$ contains the first emission contributions twice, once from the real emission quantum density $\left.\mid \rho_{m+1}^{(0)}\right)$, and once from the parton shower approximation $\left.\mathscr{H}_{I}(\tau) \mid \rho_{m}^{(0)}\right)$. The problem of double counting can be overcome if the density matrix is modified as follows

$$
\left.\mid \bar{\rho}) \equiv \mid \rho)-\int_{t_{0}}^{t_{F}} d \tau\left[\mathscr{H}_{I}(\tau)-\mathscr{V}(\tau)\right] \mid \rho_{m}^{(0)}\right)+\mathscr{O}\left(\alpha_{s}^{2}\right)
$$

Considering $\left.U\left(t_{F}, t_{0}\right) \mid \bar{\rho}\right)$ and expanding the evolution equation up to $\mathscr{O}\left(\alpha_{s}\right)$ shows that the undesired parton shower contributions are cancelled. Thus, for an infrared safe observable $F$, we can write

$$
\begin{aligned}
\bar{\sigma}[F] & =\frac{1}{m !} \int\left[d \Phi_{m}\right]\left(F\left|U\left(t_{F}, t_{0}\right)\right| \Phi_{m}\right)\left[\left(\Phi_{m} \mid \rho_{m}^{(0)}\right)+\left(\Phi_{m} \mid \rho_{m}^{(1)}\right)+\int_{t_{0}}^{t_{F}} d \tau\left(\Phi_{m}|\mathscr{V}(\tau)| \rho_{m}^{(0)}\right)\right] \\
& +\frac{1}{(m+1) !} \int\left[d \Phi_{m+1}\right]\left(F\left|U\left(t_{F}, t_{0}\right)\right| \Phi_{m+1}\right)\left[\left(\Phi_{m+1} \mid \rho_{m+1}^{(0)}\right)-\int_{t_{0}}^{t_{F}} d \tau\left(\Phi_{m+1}\left|\mathscr{H}_{I}(\tau)\right| \rho_{m}^{(0)}\right)\right],
\end{aligned}
$$

where $\Phi_{\lambda}=\left\{p, f, s^{\prime}, c^{\prime}, s, c\right\}_{\lambda}$ and $\lambda$ can be either $m$ or $m+1$. The parton shower splitting kernels are used to provide subtraction terms for the infrared singularities

$$
\begin{gathered}
\int_{t_{0}}^{\infty} d \tau \mathscr{H}_{I}(\tau)=\sum_{l} \mathbf{S}_{l} \int_{0}^{\infty} d \tau \delta\left(\tau-t_{l}\right) \Theta\left(\tau-t_{0}\right)=\sum_{l} \mathbf{S}_{l} \Theta\left(t_{l}-t_{0}\right), \\
\int_{t_{0}}^{\infty} d \tau \mathscr{V}(\tau)=\sum_{l} \int d \Gamma_{l} \mathbf{S}_{l} \Theta\left(t_{l}-t_{0}\right) \equiv \mathbf{I}\left(t_{0}\right)+\mathbf{K}\left(t_{0}\right)
\end{gathered}
$$

where the sum runs over all external legs and $\mathbf{S}_{l}$ is the total splitting kernel for a given external leg $l$. The parameter $t_{l}$ is the shower time and $\Theta\left(t_{l}-t_{0}\right)$ represents the ordering of the emissions. The phase space integration of the additional parton is denoted by $d \Gamma_{l}$. The decomposition of the integrated $\mathscr{V}(\tau)$ into two operators $\mathbf{I}\left(t_{0}\right)$ and $\mathbf{K}\left(t_{0}\right)$ is arbitrary. However, we choose $\mathbf{I}\left(t_{0}\right)$ to match the divergencies of the virtual amplitude. In addition, the limit $t_{F} \rightarrow \infty$ has been taken. This is a source of a mismatch between the fixed order and the shower calculation, however, it is numerically small due to the exponential damping by the Sudakov form factor. The total cross section, which includes parton shower evolution amounts to

$\bar{\sigma}[F]^{P S}=\frac{1}{m !} \int\left[d \Phi_{m}\right]\left(F\left|U\left(t_{F}, t_{0}\right)\right| \Phi_{m}\right)\left(\Phi_{m} \mid S\right)+\frac{1}{(m+1) !} \int\left[d \Phi_{m+1}\right]\left(F\left|U\left(t_{F}, t_{0}\right)\right| \Phi_{m+1}\right)\left(\Phi_{m+1} \mid H\right)$, 
where the following shorthands has been defined

$$
\begin{aligned}
& \left(\Phi_{m} \mid S\right) \equiv\left(\Phi_{m} \mid \rho_{m}^{(0)}\right)+\left(\Phi_{m} \mid \rho_{m}^{(1)}\right)+\left(\Phi_{m}\left|\left[\mathbf{I}\left(t_{0}\right)+\mathbf{K}\left(t_{0}\right)+\mathbf{P}\right]\right| \rho_{m}^{(0)}\right) \\
& \left(\Phi_{m+1} \mid H\right) \equiv\left(\Phi_{m+1} \mid \rho_{m+1}^{(0)}\right)-\sum_{l}\left(\Phi_{m+1}\left|\mathbf{S}_{l}\right| \rho_{m}^{(0)}\right) \Theta\left(t_{l}-t_{0}\right)
\end{aligned}
$$

The whole procedure is divided into two steps. First the samples according to Eq. (3.8) and (3.9) are generated and afterwards $U\left(t_{F}, t_{0}\right)$ is applied.

\section{Matching Exclusive Processes}

For processes with massless partons already at leading order, the matching prescription as described in the previous section must be slightly modified by the inclusion of generation cuts. The following replacements

$$
\begin{aligned}
\left(\Phi_{m} \mid S\right) & \rightarrow\left(\Phi_{m} \mid S\right) F_{I}\left(\{\hat{p}, \hat{f}\}_{m}\right), \\
\left(\Phi_{m+1} \mid H\right) & \rightarrow\left(\Phi_{m+1} \mid H\right) F_{I}\left(\{p, f\}_{m+1}\right),
\end{aligned}
$$

can be made, where $F_{I}\left(\{p, f\}_{\lambda}\right)$ is a jet function applied during the generation of events, on the momenta and flavours of $\Phi_{\lambda}$. However, when the parton shower is applied to these ensembles we can easily see that double counting is still there. Indeed, after expanding the evolution operator we obtain

$$
\begin{aligned}
\bar{\sigma}[F]^{P S} & \left.\left.\approx \frac{1}{m !} \int\left[d \Phi_{m}\right]\left(F \mid \Phi_{m}\right)\left(\Phi_{m}\left|\left[\mid \rho_{m}^{(0)}\right)+\right| \rho_{m}^{(1)}\right)+\mathbf{P} \mid \rho_{m}^{(0)}\right)\right] F_{I}\left(\{\hat{p}, \hat{f}\}_{m}\right) \\
& +\frac{1}{(m+1) !} \int\left[d \Phi_{m+1}\right]\left(F \mid \Phi_{m+1}\right)\left(\Phi_{m+1} \mid \rho_{m+1}^{(0)}\right) F_{I}\left(\{p, f\}_{m+1}\right) \\
& +\int \frac{\left[d \Phi_{m}\right]}{m !} \frac{\left[d \Phi_{m+1}\right]}{(m+1) !} \int_{t_{0}}^{t_{F}} d \tau\left(F \mid \Phi_{m+1}\right)\left(\Phi_{m+1}\left|\mathscr{H}_{I}(\tau)\right| \Phi_{m}\right) \\
& \times\left(\Phi_{m} \mid \rho_{m}^{(0)}\right)\left[F_{I}\left(\{\hat{p}, \hat{f}\}_{m}\right)-F_{I}\left(\{p, f\}_{m+1}\right)\right]+\mathscr{O}\left(\alpha_{s}^{2}\right)
\end{aligned}
$$

where the $\mathbf{I}\left(t_{0}\right)+\mathbf{K}\left(t_{0}\right)$ contribution of $\left(\Phi_{m} \mid S\right)$ has been cancelled by the linear expansion of the Sudakov form factor. This mismatch is cured by enforcing the subtraction terms to fulfill $F_{I}\left(\{\hat{p}, \hat{f}\}_{m}\right)$, i.e. by modifying the real subtracted cross section according to

$$
\left(\Phi_{m+1} \mid H\right) \longrightarrow\left(\Phi_{m+1} \mid \tilde{H}\right) \equiv\left(\Phi_{m+1} \mid \rho_{m+1}^{(0)}\right)-\sum_{l}\left(\Phi_{m+1}\left|\mathbf{S}_{l}\right| \rho_{m}^{(0)}\right) \Theta\left(t_{l}-t_{0}\right) F_{I}\left(Q_{l}\left(\{p, f\}_{m+1}\right)\right)
$$

where we make use of the inverse momentum mapping $Q_{l}$ and obtain

$$
F_{I}\left(Q_{l}\left(\{p, f\}_{m+1}\right)\right)=F_{I}\left(\{\hat{p}, \hat{f}\}_{m}\right)
$$


This modification allows us to introduce restrictions on the functional form of $F_{I}$. Expanding the shower evolution yields

$$
\begin{aligned}
\bar{\sigma}[F]^{P S} \approx & \left.\left.\frac{1}{m !} \int\left[d \Phi_{m}\right]\left(F \mid \Phi_{m}\right)\left(\Phi_{m}\left|\left[\mid \rho_{m}^{(0)}\right)+\right| \rho_{m}^{(1)}\right)+\mathbf{P} \mid \rho_{m}^{(0)}\right)\right] F_{I}\left(\{\hat{p}, \hat{f}\}_{m}\right) \\
+ & \frac{1}{(m+1) !} \int\left[d \Phi_{m+1}\right]\left(F \mid \Phi_{m+1}\right)\left(\Phi_{m+1} \mid \rho_{m+1}^{(0)}\right) F_{I}\left(\{p, f\}_{m+1}\right) \\
+ & \int \frac{\left[d \Phi_{m}\right]}{m !} \frac{\left[d \Phi_{m+1}\right]}{(m+1) !} \int_{t_{0}}^{t_{F}} d \tau\left(F \mid \Phi_{m+1}\right)\left(\Phi_{m+1}\left|\mathscr{H}_{I}(\tau)\right| \Phi_{m}\right) \\
& \times\left(\Phi_{m} \mid \rho_{m}^{(0)}\right)\left[1-F_{I}\left(\{p, f\}_{m+1}\right)\right] F_{I}\left(\{\hat{p}, \hat{f}\}_{m}\right)+\mathscr{O}\left(\alpha_{s}^{2}\right)
\end{aligned}
$$

The double counting is removed from Eq. (4.6), when the following condition is true

$$
\left[1-F_{I}\left(\{p, f\}_{m+1}\right)\right] F\left(\{p, f\}_{m+1}\right)=0 .
$$

We have used here the fact that $\left(F \mid \Phi_{m+1}\right) \sim F\left(\{p, f\}_{m+1}\right)$. This can be achieved when

$$
F_{I}\left(\{p, f\}_{m+1}\right)=1, \text { for } F\left(\{p, f\}_{m+1}\right) \neq 0 .
$$

The interpretation of the last equation is straightforward. Generation cuts $\left(F_{I}\left(\{p, f\}_{m+1}\right)\right)$ need to be more inclusive than the cuts on the final observable $\left(F\left(\{p, f\}_{m+1}\right)\right)$. Let us note here, that the MC@NLO matching formalism in combination with the Nagy-Soper parton shower introduces the intrinsic uncertainties. Let us name just the most important ones here. The Nagy-Soper parton shower treats bottom and charm quarks as massive. On the other hand in the NLO calculation we treat them as massless. Thus during the matching procedure masses for the relevant quarks are introduced by the on-shell projection. In addition, PDFs are evolved differently in the NLO calculation and in the shower. In the former case, NLO PDFs are used, whereas in the latter PDFs are evolved using Nagy-Soper splitting kernels. The evolution is, however, of higher order and the NLO accuracy is maintained if the evolutions share a common point e.g. at the low scale. Finally, the choice of the initial parton shower time $t_{0}$ in the parton evolution is arbitrary. We only require that NLO prediction is recovered for hard emissions. Thus, different choices of $t_{0}$ that fulfil this condition are possible. In consequence, the $t_{0}$ quantity should be varied to study the uncertainty of NLO+PS matching.

\section{Implementation}

The matching scheme has been implemented within the HELAC-NLO framework [1]. We have used it in conjunction with the Nagy-Soper parton shower present in DEDUCTOR version 1.0.0 [16]. Since DEDUCTOR uses spin averaged splitting functions, we only provide unpolarized event samples for showering. We supply only leading colour events even though DEDUCTOR works with the $L C+$ approximation, which comprises a full color description for collinear and soft-collinear limits and the $L C$ one for pure soft limits. The HELAC-NLO Monte Carlo program generates an event sample ready for showering with DEDUCTOR. We produce events subprocess by subprocess. First, we use HELAC-1LOOP [20] to obtain a set of unweighted leading order events with 
the virtual contributions. This set of weighted events is subsequently reweighted using HELACDIPOLES in order to include the parton shower virtual operator, which corresponds to taking into account the integrated subtraction terms. The real radiation events are generated separately with HELAC-DiPOLES. The program has been extended to provide unweighted events with positive and negative weights, which is possible, because both the real radiation and the respective subtraction weights correspond to the same phase space point. For each accepted event, we choose the most probable diagonal colour flow configuration. The generated events are stored in a Les Houches file and transferred to DEDUCTOR, which requires an on-shell projection for charm and bottom quarks and a translation of the color flow language from the LHE file to internal representation in DEDUCTOR in terms of color strings.

\section{Top quark pair production in association with a jet at NLO+PS}

We present now the results for the $p p \rightarrow t \bar{t} j$ production at the LHC with $\sqrt{s}=8 \mathrm{TeV}$. We use stable top quarks and put $m_{t}=173.5 \mathrm{GeV}$. The charm and bottom quarks are considered to be massless at fixed order. Results are obtained using the MsTw2008NLO PDF set [21] with five active flavours and the corresponding two-loop running of the strong coupling. The renormalization and factorisation scales are set to $m_{t}$, and the starting shower time to

$$
e^{-t_{0}}=\min _{i \neq j}\left\{\frac{2 p_{i} \cdot p_{j}}{\mu_{T}^{2} Q_{0}^{2}}\right\}
$$

where $p_{i}$ and $p_{j}$ are external momenta, $Q_{0}$ is the total final state momentum and $\mu_{T}=1$ for the central prediction. The anti- $k_{T}$ jet algorithm with the separation parameter $R=1$ is used to cluster partons with pseudorapidity $|\eta|<5$. The resulting jets are ordered according to their $p_{T}$. We also require the tagged jets to have transverse momentum of $p_{T}>50 \mathrm{GeV}$ and rapidity in the range of $|y|<5$. Our analysis is restricted to the perturbative parton shower evolution. Decays of unstable particles, hadronization and multiple interactions are not taken into account. The parton shower treats the charm and bottom as massive particles, thus, we use $m_{c}=1.4 \mathrm{GeV}$ and $m_{b}=4.75 \mathrm{GeV}$. Additionally, the MsTw2008NLO PDF set at $\mu_{F}=1 \mathrm{GeV}$ is provided as the starting point for the evolution in DEDUCTOR. We also use the corresponding two-loop running of $\alpha_{s}$, and restrict the parton shower to the leading colour approximation. Results presented in the following are accurate up to $\mathscr{O}\left(1 / N_{c}^{2}\right)$.

In Fig. 1 we present the transverse momentum of the $t \bar{t} j_{1}$ system together with inclusive jet cross sections. The variation bands for $\mu_{R, F}$ and $\mu_{T}$ have been obtained using the following sets of three parameter values: $\mu_{R, F}=\left\{m_{t} / 2, m_{t}, 2 m_{t}\right\}$ and $\mu_{T}=\{1 / 2,1,2\}$, respectively. The lower panels display corresponding relative deviations from the central value, separately for $\mu_{R, F}$ and $\mu_{T}$. We start the discussion with the transverse momentum of the $p_{T}\left(t \bar{t} j_{1}\right)$ system, that is given in the left panel of Fig. 1. At LO the transverse momentum of this system is zero due to momentum conservation. When NLO contributions are included, this observable diverges as the transverse momentum of the entire system goes to zero. Thus, it can only be reliably described by the fixed order calculation in the high $p_{T}$ region. The situation is changed when the parton shower is included. In that case, the low $p_{T}$ behaviour is altered strongly by the Sudakov form factor as can be seen in Fig. 1. We observe a moderate dependence on $\mu_{T}$ in the low $p_{T}$ region, up to a factor of 

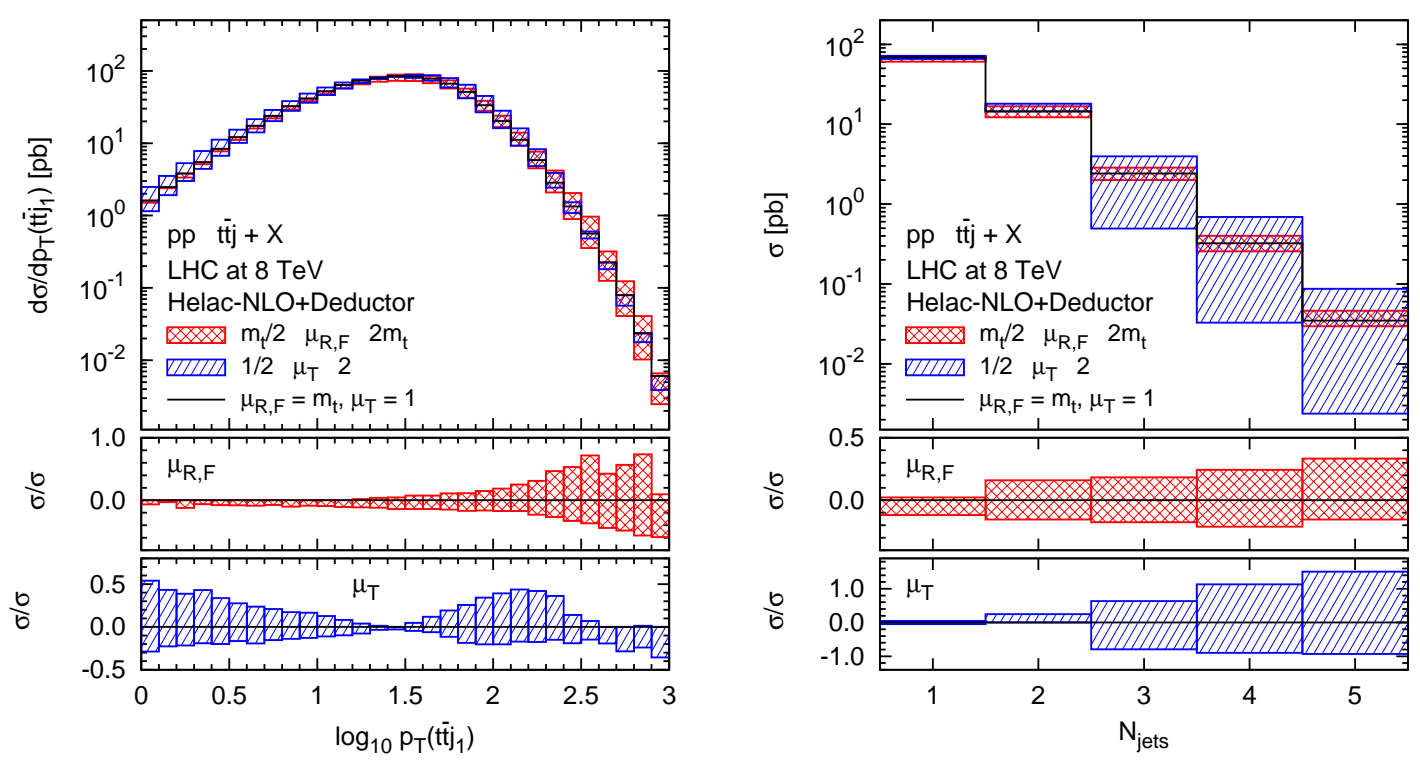

Figure 1: Differential cross section distributions as a function of the transverse momentum of the $t \bar{t} j_{1}$ system (left panel) and inclusive jet cross sections (right panel) for $p p \rightarrow t \bar{t} j+X$ at the LHC with $\sqrt{s}=8$ TeV. Results are produced by matching HELAC-NLO predictions to DEDUCTOR. The uncertainty bands depict scale and initial shower time variation. The lower panels display the corresponding relative deviation from the central value, separately for $\mu_{R, F}$ and $\mu_{T}$.

1.5 at the lower end of the spectrum. This dependence decreases down to just a few percent around $30 \mathrm{GeV}$, whereas for moderate values of $p_{T}\left(t \bar{t} j_{1}\right)$ it is at the level of $20 \%-45 \%$. The behaviour is reversed for the renormalization and factorisation scale dependence. Visible deviations from the central value occur once the matrix element dominates and grow substantially up to almost $80 \%$ at the end of the spectrum.

The inclusive jet cross sections are given in the right panel of Fig. 1. The NLO cross section with exactly one jet, which is given in the first bin, is rather insensitive to $\mu_{T}$, i.e. at the $12 \%$ level. The $\mu_{T}$ dependence is slightly larger in the second bin, where the two jet cross section, correct only at the LO level, is stored. Starting from the third bin, cross sections are described via the shower evolution alone, thus, we observe fairly large variations for both parameters, $\mu_{T}$ and $\mu_{R, F}$. For example, the scale dependence for the cross section with five jets is found to be around $35 \%$.

\section{Summary}

To summarise, we have outlined a NLO matching scheme for the Nagy-Soper parton shower. We based our construction on the original MC@NLO approach. We have presented the general formulation and a few results for top-quark pair production in association with a jet at the LHC. All results given here have been obtained using an implementation within the framework of the public codes Helac-NLO and Deductor. 


\section{Acknowledgement}

This research was supported in part by the German Research Foundation (DFG) under grant WO 1900/1-1 - Signals and Backgrounds Beyond Leading Order. Phenomenological studies for the LHC.

\section{References}

[1] G. Bevilacqua, M. Czakon, M. V. Garzelli, A. van Hameren, A. Kardos, C. G. Papadopoulos, R. Pittau and M. Worek, Comput. Phys. Commun. 184 (2013) 986 [arXiv:1110.1499 [hep-ph]].

[2] G. Bevilacqua, H. B. Hartanto, M. Kraus and M. Worek, arXiv:1509.09242 [hep-ph].

[3] P. Baernreuther, M. Czakon and A. Mitov, Phys. Rev. Lett. 109 (2012) 132001 [arXiv:1204.5201 [hep-ph]].

[4] M. Czakon, P. Fiedler and A. Mitov, Phys. Rev. Lett. 110 (2013) 252004 [arXiv:1303.6254 [hep-ph]].

[5] C. Anastasiou, C. Duhr, F. Dulat, F. Herzog and B. Mistlberger, Phys. Rev. Lett. 114 (2015) 212001 [arXiv:1503.06056 [hep-ph]].

[6] S. Frixione and B. R. Webber, JHEP 0206 (2002) 029 [hep-ph/0204244].

[7] P. Nason, JHEP 0411 (2004) 040 [hep-ph/0409146].

[8] M. L. Mangano, M. Moretti, F. Piccinini and M. Treccani, JHEP 0701 (2007) 013 [hep-ph/0611129].

[9] J. Alwall et al., Eur. Phys. J. C 53 (2008) 473 [arXiv:0706.2569 [hep-ph]].

[10] K. Hamilton, P. Nason and G. Zanderighi, JHEP 1210 (2012) 155 [arXiv:1206.3572 [hep-ph]].

[11] R. Frederix and S. Frixione, JHEP 1212 (2012) 061 [arXiv:1209.6215 [hep-ph]].

[12] S. Hoeche, F. Krauss, P. Maierhoefer, S. Pozzorini, M. Schonherr and F. Siegert, Phys. Lett. B 748 (2015) 74 [arXiv:1402.6293 [hep-ph]].

[13] S. Platzer and M. Sjodahl, JHEP 1207 (2012) 042 [arXiv:1201.0260 [hep-ph]].

[14] Z. Nagy and D. E. Soper, JHEP 0709 (2007) 114 [arXiv:0706.0017 [hep-ph]].

[15] Z. Nagy and D. E. Soper, JHEP 1206 (2012) 044 [arXiv:1202.4496 [hep-ph]].

[16] Z. Nagy and D. E. Soper, JHEP 1406 (2014) 097 [arXiv:1401.6364 [hep-ph]].

[17] M. Czakon, H. B. Hartanto, M. Kraus and M. Worek, JHEP 1506 (2015) 033 [arXiv:1502.00925 [hep-ph]].

[18] G. Bevilacqua, M. Czakon, M. Kubocz and M. Worek, JHEP 1310 (2013) 204 [arXiv:1308.5605 [hep-ph]].

[19] M. Czakon, C. G. Papadopoulos and M. Worek, JHEP 0908 (2009) 085 [arXiv:0905.0883 [hep-ph]].

[20] A. van Hameren, C. G. Papadopoulos and R. Pittau, JHEP 0909 (2009) 106 [arXiv:0903.4665 [hep-ph]].

[21] A. D. Martin, W. J. Stirling, R. S. Thorne and G. Watt, Eur. Phys. J. C 63 (2009) 189 [arXiv:0901.0002 [hep-ph]]. 